\title{
Estudio de la incidencia de ampollas en el trekking y sus factores asociados
}

\author{
Foot Blister Incidence in Trekking and Associated Factors
}

\author{
Esther Chicharro-Luna ${ }^{1}$, Domingo Orozco-Beltrán ${ }^{2}$, Salvador Sánchez Pérez ${ }^{3}$, \\ Paloma López Ros ${ }^{4}$, Nuria Padros Flores ${ }^{1}$ \\ ${ }^{1}$ Podóloga, Enfermera. Profesor colaborador. Facultad de Medicina. Universidad Miguel Hernández. \\ npadros@umh.es; ec.luna@goumh.es \\ ${ }^{2}$ Médico de familia. Profesor asociado. Dpto Salud Pública. Universidad Miguel Hernández. España. \\ dorozco@gmail.com \\ ${ }^{3}$ Médico. Profesor Titular. Dpto Psicología de la Salud. Universidad Miguel Hernández. España. \\ salva@umh.es \\ ${ }^{4}$ Podóloga. Profesor colaborador. Facultad de Medicina. Universidad Miguel Hernández \\ plopez@umh.es
}

\author{
Correspondencia: \\ Esther Chicharro Luna \\ Departamento Psicología de la Salud. Facultad de Medicina \\ Universidad Miguel Hernández. Ctra Valencia N332, km 87 \\ E-03550, Alicante, España \\ Correo-e: ec.luna@goumh.es; unpienlaluna@gmail.com
}

Fecha de recepción: 13 de Febrero de 2014

Fecha de aceptación: 23 de octubre de 2014

Los autores declaran no tener ningún tipo de interés económico o comercial.

\section{RESUMEN}

Objetivo: Determinar los factores asociados con la presencia de ampollas durante el senderismo.

Método: La población de estudio fueron 1155 peregrinos que recibieron asistencia podológica. La información fue obtenida mediante una anamnesis y una exploración podológica. Los factores analizados fueron: edad, sexo, entrenamiento previo, nacionalidad, tipo de calzado, antigüedad del mismo y kilómetros realizados.

Resultados: Las ampollas se encontraron en el 71,9 \% de los peregrinos y se distribuyeron de manera similar en ambos pies, afectando principalmente el talón (20\%) y el quinto dedo (14,6 \%). Se encontró asociación con el sexo $(\mathrm{p}<0,05)$. Las deportivas fueron las menos asociadas con ampollas $(\mathrm{p}=0,03)$. No hubo relación significativa con el entrenamiento previo y el uso de calzado nuevo.

Conclusiones: La incidencia de ampollas entre los senderistas fue del $80 \%$, y se asoció con el sexo (mayor en la mujer) y el tipo de calzado.

Palabras clave: ampolla; senderismo; trekking; camino de Santiago.

\begin{abstract}
Objective: The study objective was to determine the factors associated with presence of blisters during hiking.

Methods: The study population included 1155 pilgrims who received podiatry care. The information sources used to obtain the study variables were history by a previously devised questionnaire and foot examination. The analyzed factors were age, gender, prior training, nationality, footwear type, worn versus new shoes and kilometers hiked.

Results: Blisters were found in $71.9 \%$ of pilgrims. Blisters were distributed similarly on both feet, mostly on the heel $(20 \%)$ and the 5 th toe $(14.6 \%)$. Gender was an associated variable $(\mathrm{p}<0.05)$. The sport shoes were the least associated with presence of blisters $(\mathrm{p}=0.03)$. We found no significant relationship with prior training and use of worn shoes.

Conclusions: The incidence of blisters among hikers is almost $80 \%$, and is associated with gender (more in woman) and footwear type.

Key words: Blisters; hiking, trekking; St James’ Way.

Referencia normalizada: Chicharro-Luna, E., Orozco-Beltrán, D., Sánchez Pérez, S., López Ros, P., Padros Flores, N. Estudio de la incidencia de ampollas en el trekking y sus factores asociados. Rev. Int. Cienc. Podol. 2016; 10(1): 1-8.
\end{abstract}

Sumario: 1. Introducción, 2. Material y método, 3. Resultado, 4. Conclusiones, Bibliografía. 


\section{INTRODUCCIÓN}

El senderismo es una de las actividades deportivas más practicadas en los últimos años por su seguridad, lo que permite que pueda ser realizado por personas de diferentes edades y sin la necesidad de haber realizado un entrenamiento especial previo.

En este sentido, el Camino de Santiago (CdS) es quizá uno de los caminos de peregrinación más conocidos. Se trata de una ruta que finaliza en Santiago de Compostela (España), donde se encuentran las reliquias del Apóstol Santiago el Mayor. Durante la Edad Media fue un camino muy concurrido y actualmente vuelve de nuevo a estar en auge, acogiendo cada año a más de 160.000 senderistas de todas las edades y nacionalidades. Existe gran variabilidad tanto en los motivos de su realización (deportivos, religiosos, sociales), como en la duración del mismo (días a semanas) o en la preparación previa de los caminantes.

La mayoría de las lesiones que se producen durante la práctica del senderismo se localizan en los miembros inferiores, pie, tobillo y rodilla, asociadas a un uso excesivo de los mis$\operatorname{mos}^{1}$. Los pies, quizá, son la parte del cuerpo que se ve más afectada por el sobreesfuerzo al que el caminante se ve sometido, destacando las ampollas como la lesión con mayor incidencia en este deporte.

Las ampollas por fricción aparecen con frecuencia al iniciar un deporte o al aumentar una actividad. No suelen causar problemas médicos significativos aunque son bastante dolorosas y pueden dificultar la finalización del senderismo, provocando una marcha antiálgica que puede repercutir negativamente en otras articulaciones más proximales como la rodilla o la cadera ${ }^{2}$. El objetivo que nos planteamos es determinar la prevalencia y los factores asociados a la presencia de ampolla entre los peregrinos que realizan senderismo en el Camino de Santiago.

\section{MATERIAL Y MÉTODO}

La población objeto de estudio está constituida por 1155 peregrinos que recibieron asistencia podológica en varios albergues de León, durante el periodo del 16 de Julio al 18 de Agosto de 2011. Las fuentes de información para la obtención de las variables de estudio fueron la anamnesis mediante una encuesta previamente confeccionada y la exploración podológica.

Los factores analizados fueron: edad, sexo, entrenamiento previo, nacionalidad, tipo de calzado, antigüedad del mismo y kilómetros realizados. El entrenamiento previo se valoró como positivo si la respuesta era afirmativa a la pregunta ¿Ha realizado entrenamiento previo para hacer el CdS? aceptando cualquier duración y modalidad. El tipo de calzado se categorizó en Bota treking; Zapatilla treking; Sandalia treking y zapatillas deportivas. Se consideró un calzado nuevo aquel que tenía una duración menor de tres meses. Los kilómetros realizados se calcularon a posteriori midiendo la distancia desde el punto de partida indicado por el peregrino, hasta el lugar de atención podológica.

El trabajo de campo ha sido desarrollado por profesionales Podólogos y entrenados en la detección y tratamiento de lesiones podológicas.

Los datos recogidos han sido codificados para su tratamiento estadístico, tabulados y mecanizados informáticamente, analizándolos mediante el paquete de programas informáticos SPSS para Windows, versión 17. Se ha realizado un análisis descriptivo y se ha calculado las proporciones para variables cualitativas y las medidas de tendencia central y dispersión para cuantitativas. En el análisis bivariante se utilizó la prueba $t$ de Student para variables cuantitativas con cualitativas de 2 categorías y ANOVA si presentaba más de 2 categorías. Finalmente se realizó un análisis multivariante para aislar posibles factores de confusión y conocer el grado de explicación del modelo.

\section{RESULTADOS}

Los senderistas tenían una edad media de 37.60 con un rango comprendido entre 9 y 78 años, siendo un $48.7 \%$ mujeres y un $51.3 \%$ varones.

Sólo un $28.1 \%$ de los peregrinos no presentó ampollas frente al $71.9 \%$ que sí sufrió esta lesión, de los cuales el $23.4 \%$ tuvo una lesión ampollosa y el $48.6 \%$ dos o más lesiones. La distribución de las ampollas fue similar en am- 
bos pies, localizándose con mayor frecuencia en el talón (20\%) y en el $5^{\circ}$ dedo (un $14.6 \%$ ).

En relación a la edad, se observa una tendencia a aumentar el número de lesiones ampollosas al disminuir la edad del paciente, con una edad media de 38.8 años los que no presentaban lesión y 35.26 años los que presentaban tres o más ampollas. $(\mathrm{p}=0.13)$ (Figura 1).

El sexo es una variable asociada, encontrando que presentaron ampollas un $74.2 \%$ de las mujeres, frente a un $68.9 \%$ de los hombres. $(\mathrm{p}<0.05)$ (Figura 2).
Respecto al entrenamiento previo a la actividad, un $48.78 \%$ no había realizado ninguna actividad deportiva previa durante el último mes, frente al 51.1\% que sí dijo haberlo hecho. No se encontraron diferencias significativas en la presencia de lesiones ampollosas entre los que habían entrenado $(71.4 \%)$ y los que no lo habían hecho $(71.8 \%)(\mathrm{p}=0.87)$ (Figura 3$)$.

Respecto a la nacionalidad el $52.9 \%$ de los peregrinos atendidos eran de nacionalidad española, seguidos de italianos (12.6\%), alemanes $(7.9 \%)$ y franceses $(4.9 \%)$. Se observó que aunque efectivamente los europeos suelen co-

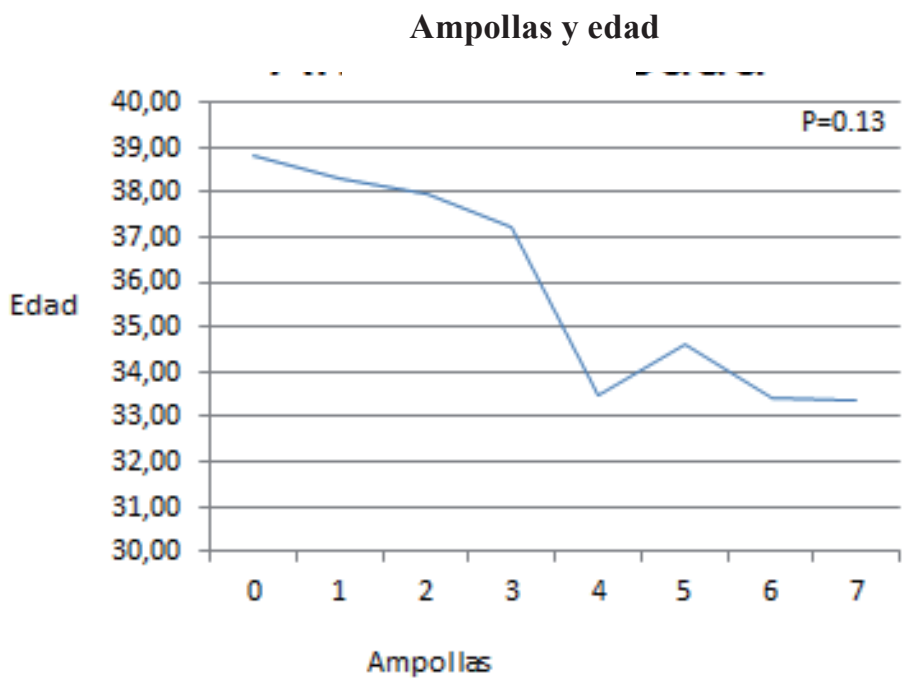

Figura 1. Relación entre ampollas y edad.

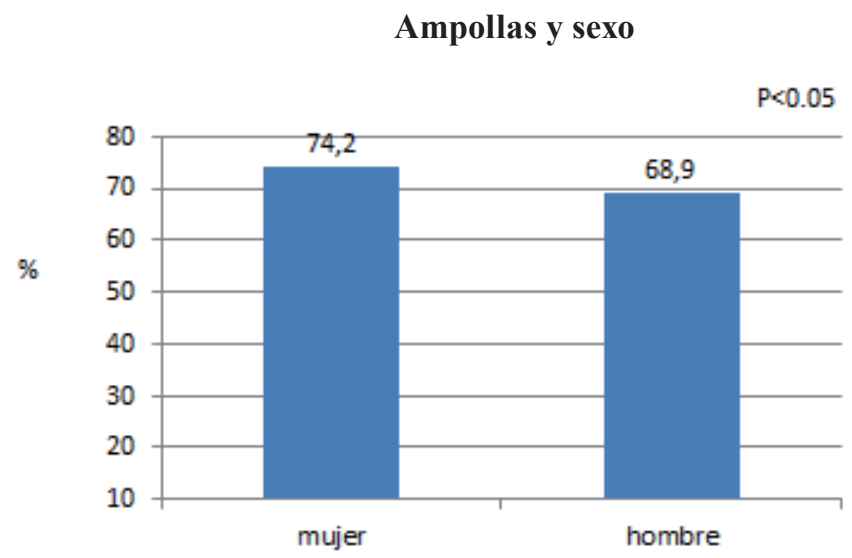

Figura 2. Relación entre ampollas y sexo. 
menzar el camino en la zona pirenaica, no había una relación directa con la presencia de este tipo de lesiones, encontrando afectación en un $78.9 \%$ de los alemanes, $73.4 \%$ de los italianos, $69.9 \%$ de los españoles y $73.4 \%$ de los franceses $(\mathrm{p}=0.18)$ (Figura 4).

Un $32.4 \%$ de los peregrinos estrenaron calzado para realizar el camino, frente a un $67.4 \%$ que utilizaron calzado ya usado, no encontrando diferencias significativas con la presencia de ampollas $(\mathrm{p}=0.12)$ (Figura 5).

Respecto al tipo de calzado, un 54.2\% utilizaban bota de treking, un $18.3 \%$ zapatilla de- portiva, el $11.9 \%$ zapatilla de treking, un 5.4\% sandalia treking y un $8 \%$ alterna varios tipos de calzado durante el recorrido (Figura 6).

De todos los calzados utilizados, la zapatilla deportiva es la que menos se asoció a la presencia de ampollas $(p=0.03)$. Es importante resaltar que las ampollas del $5^{\circ}$ dedo (una de las lesiones ampollosas más frecuentes) se asoció en un mayor porcentaje a botas de treking y en un menor porcentaje, tanto en el pie derecho como en el izquierdo, a calzado deportivo (Figura 7).

La media de kilómetros en los pacientes con ampollas era de $267.6 \mathrm{~km}$. Encontramos

\section{Ampollas y entrenamiento}

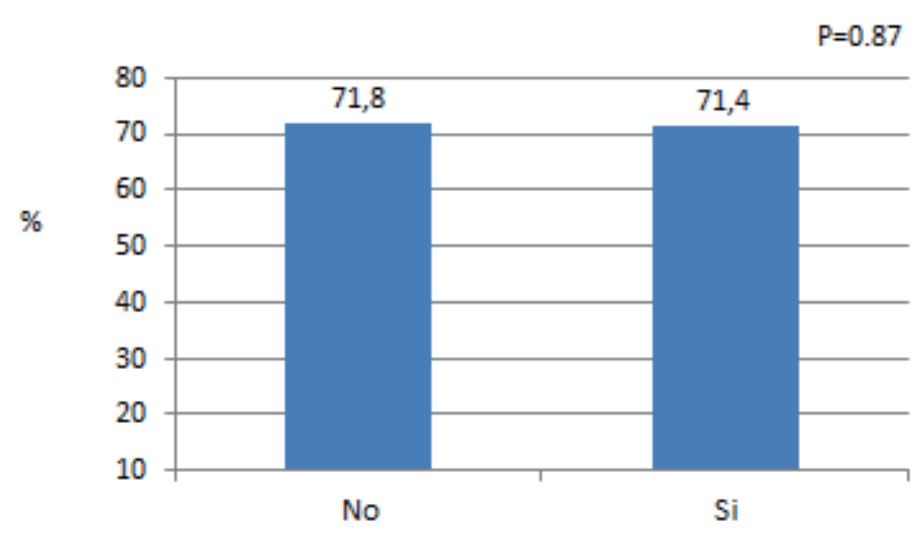

Figura 3. Relación entre ampollas y entrenamiento previo.

\section{Ampollas y país de procedencia}

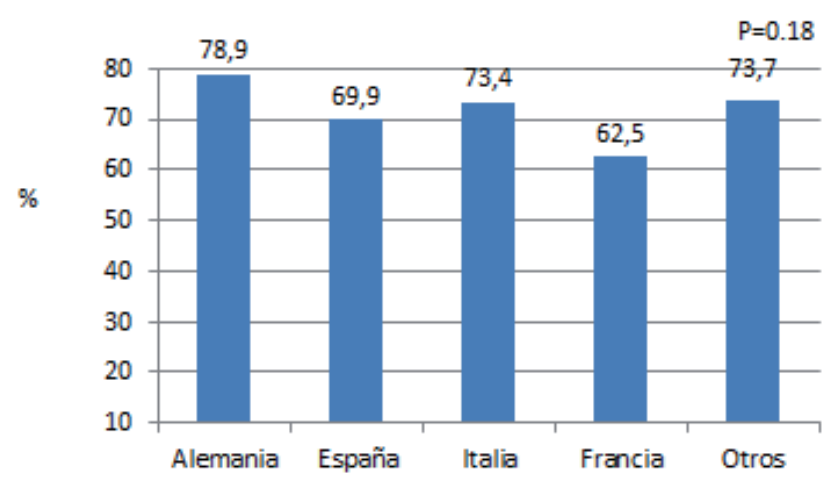

Figura 4. Relación entre ampollas y nacionalidad. 


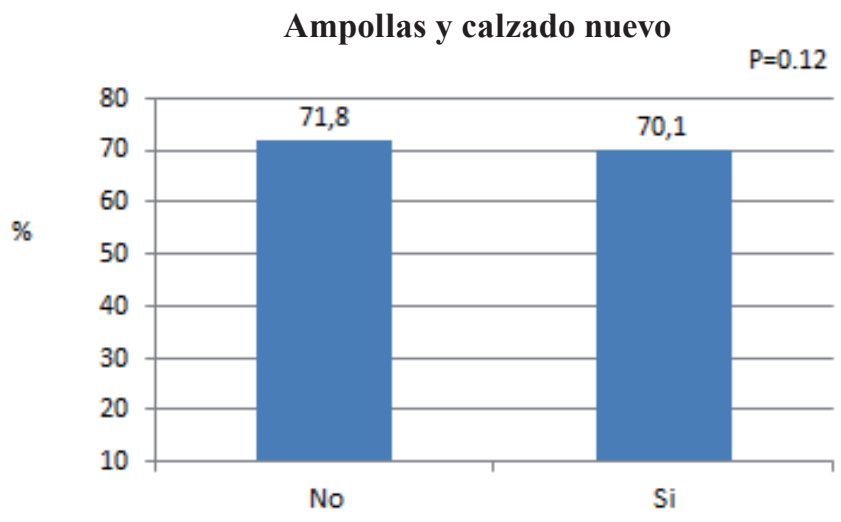

Figura 5. Relación entre ampollas y el uso de un calzado nuevo.

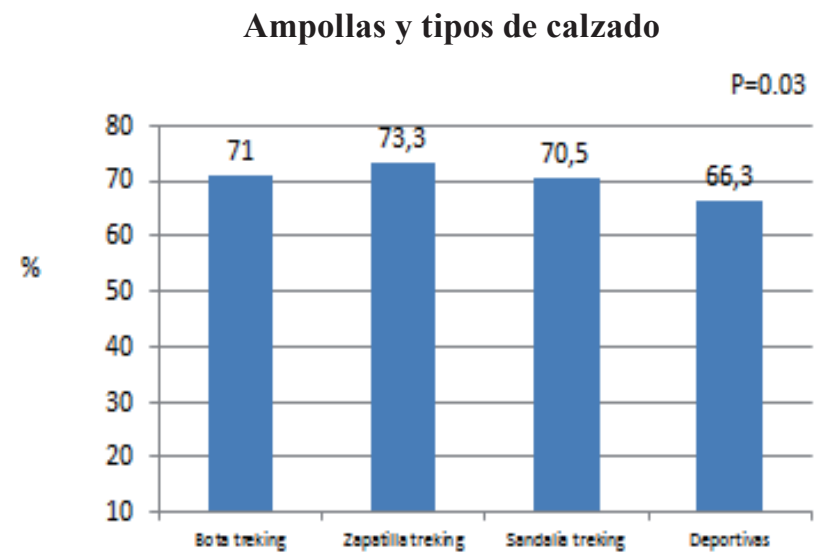

Figura 6. Relación entre ampollas y el tipo de calzado.

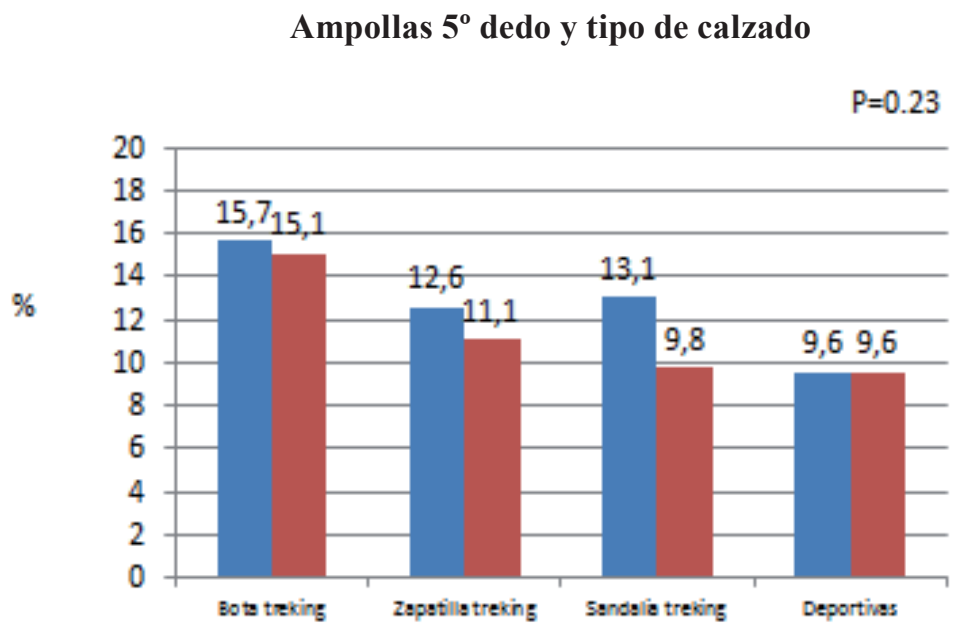

Figura 7. Localización de las ampollas. 
que el número de ampollas aumentaba a medida que lo hacían los kilómetros realizados, de manera que los pacientes que tenían una lesión habían andado una media de $269.64 \mathrm{~km}$ y los que tenían seis lesiones $483.62 \mathrm{~km}(\mathrm{p}=0.00)$ (Figura 8).
Al realizar el análisis multivariante el modelo explica un $12 \%$ de la variabilidad de la variable dependiente (existencia de ampolla), la única variable que mostró asociación fue el sexo no encontrando asociación significativa en el resto de variables (Tabla 1).

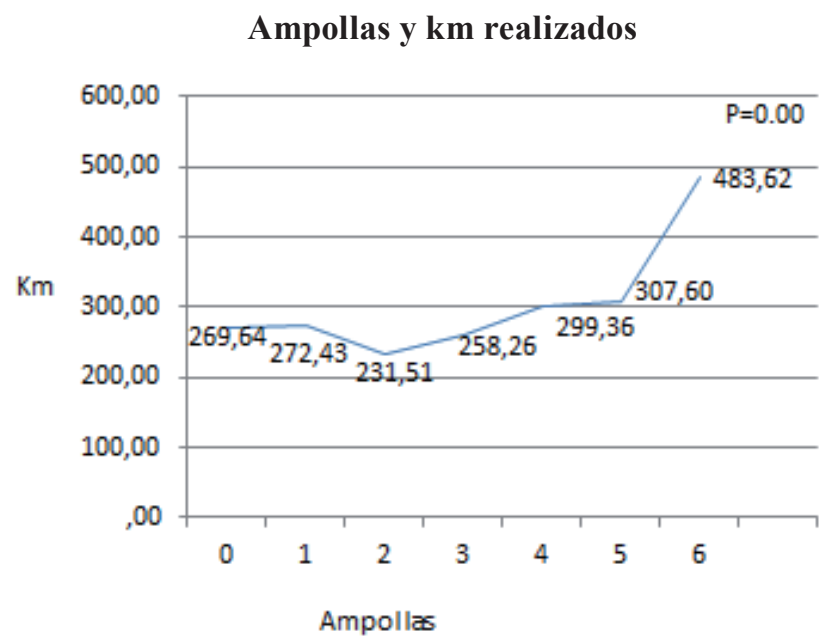

Figura 8. Relación entre ampollas y kilómetros realizados.

Tabla 1. Análisis multivariante.

\begin{tabular}{|l|r|r|r|}
\hline & Resumen del modelo \\
\hline Paso & $\begin{array}{c}-2 \text { log de la } \\
\text { verosimilitud }\end{array}$ & $\begin{array}{c}\text { R cuadrado } \\
\text { de Coxy Snell }\end{array}$ & $\begin{array}{c}\text { R cuadrado } \\
\text { de } \\
\text { Nagelkerke }\end{array}$ \\
\hline 1 & $1331,546^{\text {a }}$ &, 009 &, 012 \\
\hline
\end{tabular}

a. La estimación ha finalizado en el número de iteración 4 porque las estimaciones de los

parámetros han cambiado en menos de ,001.

\begin{tabular}{|c|c|c|c|c|c|c|c|c|c|}
\hline \multicolumn{10}{|c|}{ Variables en la ecuación } \\
\hline & & \multirow[b]{2}{*}{$\mathrm{B}$} & \multirow[b]{2}{*}{ E.T. } & \multirow[b]{2}{*}{ Wald } & \multirow[b]{2}{*}{$\mathrm{gl}$} & \multirow[b]{2}{*}{ Sig. } & \multirow[b]{2}{*}{$\operatorname{Exp}(B)$} & \multicolumn{2}{|c|}{ I.C. $95 \%$ para EXP(B) } \\
\hline & & & & & & & & Inferior & Superior \\
\hline \multirow[t]{8}{*}{ Paso $1^{a}$} & Edad &,- 004 &, 003 & 1,281 & 1 & ,258 &, 996 &, 990 & 1,003 \\
\hline & Sexo &,- 296 & ,135 & 4,780 & 1 & 029 & ,744 &, 571 & ,970 \\
\hline & Kilometros &, 000 &, 000 &, 005 & 1 & ,945 & 1,000 & ,999 & 1,001 \\
\hline & Calzado & 043 & ,036 & 1,361 & 1 & ,243 & 1,043 & ,971 & 1,121 \\
\hline & Tiempocalzado &,- 218 & , 146 & 2,236 & 1 & ,135 &, 804 & ,604 & 1,070 \\
\hline & Entrenamiento_deporte &, 072 & 134 & ,284 & 1 & ,594 & 1,074 & ,826 & 1,397 \\
\hline & pais 5 categorias &, 001 & ,053 &, 000 & 1 & ,983 & 1,001 & ,902 & 1,111 \\
\hline & Constante & 1,296 & ,251 & 26,702 & 1 &, 000 & 3,655 & & \\
\hline
\end{tabular}

a. Variable(s) introducida(s) en el paso 1: Edad, Sexo, Kilometros, Calzado, Tiempocalzado, Entrenamiento_deporte, pais 5 categorias. 


\section{DISCUSIÓN}

En el estudio de Bulware en 334 personas que caminaron el sendero de los Apalaches durante al menos 7 días, destacan las ampollas de los pies $(64 \%, \mathrm{n}=180)$, la irritación de la piel $(51 \%, \mathrm{n}=143)$, y el dolor articular $(36 \%, \mathrm{n}=$ 102) como lo problemas médicos más comunes ${ }^{3}$. Granero en su estudio destaca también las ampollas en los pies (51\%) como el problema físico más sufrido por los peregrinos en su recorrido a Santiago, seguido de las llagas y las tendinitis por sobresfuerzo $(38.6 \%)$. Lesiones como esguinces o rozaduras, se presentan como puntuales con una baja incidencia entre los excursionistas ${ }^{4}$. Estos resultados coinciden con los obtenidos en este estudio, donde un $71.9 \%$ de los peregrinos fue atendido por presentar ampollas en diferentes localizaciones de los pies.

La relación entre la menor edad y la aparición de ampollas puede explicarse por varios aspectos, en primer lugar debemos tener presente que las ampollas están altamente relacionadas según algunos estudios ${ }^{5}$ con una marcada hiperhidrosis, alteración más frecuente en pacientes jóvenes. En segundo lugar, son los peregrinos de menor edad los que realizan mayor número de etapas y más largas, lo que podría contribuir a una mayor incidencia de ampollas en el pie.

Al igual que en el estudio de Granero, hemos encontrado que muy pocos senderistas habían realizado entrenamiento previo a la actividad, siendo probablemente uno de los factores que inciden en la presencia de tan alto porcentaje de lesiones podológicas. Los resultados del análisis coinciden con los expuestos por este autor, quien indica en su estudio que el $42 \%$ de los excursionistas no había realizado una preparación física anterior ${ }^{6}$. En nuestro estudio un $48.78 \%$ no habían realizado ninguna actividad deportiva previa durante el último mes, frente al $51.1 \%$ que sí dice haberlo hecho. Cabe destacar a este respecto que, a pesar de que autores como Bravo ${ }^{7}$ o Vázquez et. al ${ }^{8}$ recomiendan realizar un entrenamiento basado en largas caminatas y excursiones por el monte, un gran porcentaje de los peregrinos entrevistados entrena realizando actividades deportivas como running, natación o incluso artes marciales.
La falta de asociación de entrenamiento previo como prevención en la presencia de ampollas, puede ser debido a que las lesiones dérmicas están más relacionadas con el tipo de calzado y calcetín utilizado, así como el comportamiento biomecánico del pie durante la marcha, más que con el entrenamiento previo, que puede ser más determinante cuando hablamos de lesiones tendinosas o musculares. Además, en el estudio existe una limitación, ya que se registró el tipo de entrenamiento realizado en el último mes, pero no la frecuencia semanal del mismo.

Granero indica que la nacionalidad está relacionada con la presencia de ampollas en los pies, haciendo referencia a que los europeos tienden a iniciar la peregrinación en lugares más alejados como Saint Pied de Port o Roncesvalles, a diferencia de los españoles que comienzan en puntos más cercanos a Santiago de Compostela ${ }^{4}$. En nuestro estudio hemos observado que aunque efectivamente los europeos suelen comenzar el camino en la zona pirenaica, no existe una relación directa entre la nacionalidad y la presencia de esta lesión.

Hay pocos estudios que relacionen la asociación entre el tipo de calzado y las lesiones encontradas durante el senderismo. Sin embargo, es importante que el calzado sea el adecuado para cada práctica deportiva, teniendo en cuenta el gesto deportivo y el tipo de terreno donde se vaya a desarrollar ésta. Anderson, tras analizar 128 senderistas en su estudio, concluye que el calzado no parece estar asociado a la presencia de parestesias o lesiones músculo esqueléticas ${ }^{9}$. Otros autores creen que la elección correcta del calzado es fundamental para prevenir la aparición de lesiones podológicas durante la marcha.

El tipo de calzado adecuado depende de las características del terreno por el que se realice la marcha. Habitualmente se recomienda calzado con suela de poliuretano, como la zapatilla deportiva, para absorber impactos cuando se camina por asfalto y botas de montaña con suela de mayor agarre y contención de tobillo, cuando se camina por terreno irregular montañoso, con el fin de evitar caídas y esguinces

De todos los calzados utilizados, la zapatilla deportiva es la que menos se asociaba a la presencia de ampollas, pudiendo concluir que se 
trata de un calzado adecuado para este tipo de senderismo durante la época estival, momento en el que se realizó el estudio. Es importante resaltar que las ampollas del $5^{\circ}$ dedo (una de las lesiones ampollosas más frecuentes) se asoció en un mayor porcentaje a botas de treking $\mathrm{y}$ en un menor porcentaje al calzado deportivo.

En este sentido, cabe indicar que el puesto de atención podológica y de recogida de datos se situaba en la provincia de León, siendo hasta allí el tipo de camino mayoritariamente asfaltado. Quizá este sea el motivo por el que el uso de deportivas, con una mayor absorción de impactos en carretera, haya sido asociado a un menor número de lesiones. Sería necesario realizar estudios que recogiesen también etapas de terreno montañoso.

En lo que sí están prácticamente de acuerdo todos los autores es en la necesidad de utilizar un calzado usado anteriormente que haya sido adaptado al pie. Sorprendentemente, no hay diferencias importantes en la presencia de ampollas entre los que utilizaron un calzado nuevo y los que no. Encontramos a este respecto una limitación, ya que no se valoró la frecuencia de uso del calzado usado, únicamente se tuvo en cuenta que tuviese una antigüedad mayor de tres meses.
Otro factor importante a tener en cuenta es el número de kilómetros realizados. La media de kilómetros en los pacientes con ampollas era de $267.6 \mathrm{~km}$. Como era de esperar, se observó que el número de ampollas encontradas aumentaba a medida que lo hacían los kilómetros realizados, de manera que los pacientes que tenían una lesión habían andado una media de $269.64 \mathrm{~km}$ y los que tenían seis lesiones $483.62 \mathrm{~km}$.

El estudio tiene algunas limitaciones ya que no fue valorado el calcetín, un elemento íntimamente relacionado con el pie, que según el estudio de Van Tiggelen, influye de forma decisiva en la presencia de ampollas ${ }^{1}$. Sería interesante valorar en próximos estudios el tipo de calcetín usado y la frecuencia de cambio de los mismos, como posible factor asociado a lesiones podológicas.

\section{CONCLUSIÓN}

La incidencia de ampollas durante el senderismo es casi del $80 \%$, y se asocia con el sexo (mayor incidencia en mujeres) y el tipo de calzado, pero no con la utilización de calzado nuevo o entrenamiento previo.

\section{BIBLIOGRAFÍA}

1. Blake RL, Ferguson HJ. Walking and hiking injuries. A one year follow-up study. J Am Podiatr Med Assoc 1993; 83(9):499-503.

2. Van Tiggelen D, Wickes S, Coorevits P, Dumalin M, Witvrouw E. Sock systems to prevent foot blisters and the impact on overuse injuries of the knee joint. Mil Med 2009; 174(2):183-189.

3. Boulware DR, Forgey WW, Martin II WJ. Medical risks of wilderness hiking. Am J Med 2003; 114(4):288-293.

4. Granero Gallegos A., Ruiz Juan F., García Montes ME. Lesiones y problemas físicos padecidos por senderistas y cicloturistas en una ruta por etapas. El caso del camino de Santiago. Rev int med cienc act fis deporte 2006; 22:99.

5. Knapik JJ, Reynolds K, Barson J. Influence of an antiperspirant on foot blister incidence during crosscountry hiking. J Am Acad Dermatol 1998; 39(2 I):202-206.

6. Antonio Granero Gallegos, Francisco Ruiz Juan, $M^{a}$ Elena García Montes, Gabriel Flores Allende. Estudio de la preparación física realizada para recorrer el Camino de Santiago. Retos. Nuevas tendencias en Educación Física, Deporte y Recreación 2007(11):60-65.

7. Bravo, M. Guía práctica del peregrino. El Camino de Santiago. 5a ed. León: Everest; 1998

8. Vázquez J, Solana MR, Quintas C. Consejos médicos para el peregrino. Barcelona: Paidotribo; 2004

9. Anderson Jr. LS, Rebholz CM, White LF, Mitchell P, Curcio III EP, Feldman JA, et al. The impact of footwear and packweight on injury and illness among long-distance hikers. Wilderness and Environmental Medicine 2009; 20(3):250-256. 